\title{
Prenatal findings in congenital leukemia: a case report.
}

\section{AUTHOR(S):}

Sato, Yukiyasu; Izumi, Yukiko; Minegishi, Katsura; Komada, Munekazu; Yamada, Shigehito; Kakui, Kazuyo; Tatsumi, Keiji; Mikami, Yoshiki; Fujiwara, Hiroshi; Konishi, Ikuo

\section{CITATION:}

Sato, Yukiyasu ...[et al]. Prenatal findings in congenital leukemia: a case report.. Fetal diagnosis and therapy 2011, 29(4): 325-330

\section{ISSUE DATE:}

2011-02-05

URL:

http://hdl.handle.net/2433/197590

\section{RIGHT:}

(c) 2011 S. Karger AG, Basel; This is not the published version. Please cite only the published version.; この論文は出版社版でありません。引用の 際には出版社版をご確認ご利用ください。 
1 Prenatal Findings in Congenital Leukemia: A Case Report

2

3 Yukiyasu Sato ${ }^{1 *}$, Yukiko Izumi $^{1}$, Katsura Minegishi ${ }^{2}$, Munekazu Komada ${ }^{2}$, Shigehito

4 Yamada $^{2}$, Kazuyo Kakui ${ }^{1}$, Keiji Tatsumi ${ }^{1}$, Yoshiki Mikami ${ }^{3}$, Hiroshi Fujiwara ${ }^{1}$, and $5 \quad$ Ikuo Konishi ${ }^{1}$

6

$7 \quad{ }^{1}$ Department of Gynecology and Obstetrics and ${ }^{2}$ Congenital Anomaly Research Center, 8 Kyoto University Graduate School of Medicine

$9 \quad{ }^{3}$ Department of Diagnostic Pathology, Kyoto University Hospital 10

11 *Address correspondence and reprint requests to: Yukiyasu Sato, M.D., Ph.D.

12 Department of Gynecology and Obstetrics, Kyoto University Graduate School of 13 Medicine, Sakyo-ku, Kyoto 606-8507, Japan.

14 Tel; 81-75-751-3269: Fax; 81-75-761-3967: E-mail; yukiyasu@kuhp.kyoto-u.ac.jp 15

16 Short title; Fetal Leukemia

17 
1 Established facts

2 - Neonatal leukemia has poor prognosis.

3 - Neonatal leukemia associated with Down syndrome has a natural tendency to

4 undergo spontaneous remissions and reported mortality rate is much lower than that

5 without Down syndrome.

6 - Significant number of neonatal leukemia arises in utero and fetal leukemia should

7 be kept in mind as an important cause of intrauterine deterioration and death.

8 - Hepatosplenomegaly and hydrops fetalis are two characteristic prenatal features of 9 congenital leukemia.

10

11 Novel Insights

12 - Prenatal course of leukemic fetus including sequential changes in the 13 ultrasonographic findings, pulse Doppler indices, and fetal heart rate patterns is 14 specified for the first time.

15 - Experience in our case suggests elevation of pulsatility index of the umbilical artery 16 with paradoxically enlarged fetal trunk as an early sign of fetal leukemia.

17 - Experiences in our case and in the past reported cases suggest two distinct 18 mechanisms for hydrops development caused by fetal leukemia. The first is 19 leukemic cell involvement of multiple fetal organs including placenta and the second 20 is chronic circulatory insufficiency due to hyperviscosity attributable to leukocytosis.

21 - Prenatal diagnoses of fetal leukemia have been reported in only 10 cases, all of 22 which were associated with trisomy 21. This suggests that leukemic fetuses 23 associated with Down syndrome are likely to have a milder prenatal course than those 24 without Down syndrome, thus giving a time for cordocentesis. 
1 Prenatal Findings in Congenital Leukemia: A Case Report

2

3 Abstract

4

5 We here describe a case of congenital leukemia that ended in intrauterine fetal demise

6 at 30 weeks of gestation. Acute enlargement of the fetal trunk, elevated pulsatility

7 index of the umbilical artery with concomitant decline of pulsatility index of middle

8 cerebral artery, pleural effusion, and polyhydramnios preceded the fetal death.

9 Diagnosis of congenital myeloid leukemia was suggested by microscopic examination

10 of the placental tissue, revealing immature myeloid precursors filling the lumina of fetal 11 vessels in the umbilical cord and chorionic villi. Extensive vascular involvement of 12 the placenta by leukemic cells was considered to be a primary cause of the fetal death. 13

14 Keywords; Cordocentesis, Doppler velocimetry, Down syndrome, Hydrops fetalis, 15 Intrauterine death, Hepatosplenomegaly 
1 Introduction

2

3 Neonatal leukemia, which occurs at a rate of 1 per 5 million births, is the leading 4 cause of neonatal death due to neoplastic disease [1]. Recent molecular studies 5 indicate that significant number of neonatal leukemia arises in utero [2]. In this 6 respect, fetal leukemia should be kept in mind as an important cause of intrauterine 7 deterioration and death. However, prenatal history of congenital leukemia has not 8 been well characterized. Herein, we describe a case of congenital leukemia where 9 acute enlargement of the fetal trunk, elevated pulsatility index of the umbilical artery 10 with concomitant decline of pulsatility index of the middle cerebral artery, pleural 11 effusion, and polyhydramnios were noted before fetal demise at 30 weeks of gestation. 12 
Case Report

A 35-year-old woman (gravida 0, prara 0) at $27^{+5}$ weeks' gestation was referred and admitted to our hospital due to moderate amount of vaginal bleeding from total placenta previa. She was taking antipsychotic medication for schizophrenic disorder. Her blood screening was negative for irregular antibody or treponema pallidum. Recent infection with rubella was also excluded.

After hospitalization, bleeding gradually decreased and disappeared with bed rest and tocolytic administration. On admission, fetal measurements of biparietal diameter (BPD), fetal trunk area (FTA), and femur length (FL) were all within the normal range. No fetal malformation was noted and amniotic fluid volume was normal. Pulsatility indices of umbilical artery (UmA-PI) and middle cerebral artery (MCA-PI) measured with Doppler ultrasonography (Voluson730Pro; GE Healthcare, Austria) were all within the normal level (Figure 1A and B). Fetal heart rate (FHR) monitoring showed reassuring pattern.

At $29^{+6}$ weeks' gestation, UmA-PI was elevated beyond the normal limit (Figure 1A). MCA-PI was decreased but remained within the normal range (Figure 1B). Although BPD and FL was within the normal limits, FTA was abruptly enlarged to $64.29 \mathrm{~cm}^{2}$ (+2.3SD). FHR monitoring showed reassuring pattern.

At $30^{+2}$ weeks' gestation, the patient felt diminished fetal movement. Ultrasonography showed polyhydramnios (Figure 1C) and mild fetal pleural effusion (Figure 1D). Further elevation of UmA-PI and decline of MCA-PI was noted (Figure $1 \mathrm{~A}$ and $\mathrm{B})$. Doppler waveform of bilateral uterine artery was normal. FTA was increased to $68.23 \mathrm{~cm}^{2}$ (+2.6SD). FHR monitoring exhibited decreased beat-to-beat variability (Figure 2A).

At $30^{+3}$ weeks' gestation, FHR monitoring showed small dip-like deceleration observed on every uterine contraction, which disappeared after tocolysis (Figure 2B). UmA-PI was further elevated and MCA-PI fell below the normal limit (Figure 1A and B). Doppler waveforms of the inferior vena cava and ductus venosus were normal. Anticipating the need of expeditious premature delivery, maternal betamethasone was administered for the prevention of neonatal respiratory distress syndrome. Three hours later, mild bradycardia (100-110 beat per minute) with loss of variability was noted and fetal heartbeat suddenly became undetectable (Figure 2C). Ultrasonography 
1 confirmed the fetal death.

2 Two days later, a male stillborn weighing $1872 \mathrm{~g}$ (+1.3SD) was delivered with

3 cesarean section indicated for total placenta previa. The newborn was macerated, but

4 no apparent external malformation was noted. The parents declined to have the

5 autopsy. Postpartum studies on the maternal serum excluded recent infection with

6 cytomegalovirus, parvovirus, herpes simplex, or toxoplasma.

7 Grossly, the placenta (410 g in weight) appeared normal. Umbilical cord (24 cm in

8 length) was inserted into the central portion of the placental disc without any sign of

9 stricture or overcoiling. The fetal surface of the placenta was translucent.

10 Microscopically, numerous small- to medium-sized atypical immature-looking cells

11 with high nuclear to cytoplasmic ratio were observed in the fetal vessels including

12 intravillous capillaries as well as medium- to large-sized veins and arteries in the

13 chorionic plate and the umbilical cord (Figure 3A, B and C). On the other hand,

14 maternal vessels in the decidual tissue and intervillous spaces were free of

15 above-mentioned atypical cells. In some areas, intervillous fibrin deposition and

16 thrombosis was identified. Immunohistochemically, these atypical cells were positive

17 for myeloperoxidase (Figure 3D) and negative for CD3 (T-cell marker) and CD20

18 (B-cell marker), indicating the myeloid lineage of differentiation. The Ki-67 (MIB-1)

19 labeling index was up to 50\% (Figure 3E). This constellation of findings suggested

20 the diagnosis of acute myeloid leukemia of the fetus. 


\section{Discussion}

In the current case, cause of the sudden fetal deterioration and death had been totally unknown until the placental pathology was reported. Possible causes for fetal pleural effusion associated with polyhydramnios such as cardiopulmonary anomaly, congenital infection, and erythroblastosis fetalis were excluded by the fetal ultrasonography and maternal blood tests. Numerous atypical myeloperoxidase-positive cells in the fetal vessels of the placenta and the umbilical cord suggested acute myeloid leukemia of the fetus. It is known that fetus and neonate are liable to produce leukemoid reaction in response to congenital infection or eryhtroblastosis fetalis [3]. Although the placental pathology could also be explained by the leukemoid reaction, exclusion of congenital infection and eryhtroblastosis fetalis by maternal blood tests convinced us of the diagnosis of fetal leukemia.

The FTA became abnormally large after $29^{+6}$ weeks' gestation, suggesting the development of fetal hepatosplenomegaly. Abrupt elevation of UmA-PI can be explained by occlusion of the placental and the umbilical vessels by leukemic cells, as revealed by microscopic examination. Polyhydramnios could be caused by increase of exudates from the infiltrated placental villi. Subsequent decline of MCA-PI indicated that the blood flow redistribution towards the fetal brain took place as a compensating mechanism. Since no cardiac anomaly was detected by fetal echocardiography and Doppler waveforms of inferior vena cava and ductus venosus were normal immediately prior to fetal death, the pleural effusion was considered to be due to pleural infiltration by leukemic cells rather than acute cardiac failure. Loss of variability in FHR tracing followed by dip-like deceleration concurrent with uterine contraction and prolonged bradycardia might reflect fetal hypoxia caused by impaired oxygen supply to the fetus via the occluded umbilical vein. Finally, fetal metabolic acidosis resulting from progressive hypoxia is considered to be a cause of sudden cardiac arrest. Therefore, acute circulatory failure due to placental vessel occlusion by leukemic cells is likely to be a primary cause of the sudden fetal demise.

Hepatosplenomegaly and hydrops fetalis are two characteristic prenatal features of a leukemic fetus [4-6] [7-9]. Since extramedullary hematopoiesis physiologically occurs in liver and spleen during intrauterine period, fetuses easily develop hepatosplenomegaly in response to various hematological disorders including leukemia. 
1 Hepatosplenomegaly is often revealed as abnormally enlarged FTA. In clinical

2 practice, elevation of UmA-PI is most often seen in chronic uteroplacental insufficiency

3 that is usually associated with restricted FTA growth. Thus, elevation of UmA-PI with

4 paradoxically enlarged FTA, as seen in the present case, may be an early sign of fetal 5 leukemia.

6 Hydrops fetalis is generally associated with poor prognosis [10]. Two distinct 7 mechanisms could be considered for hydrops development caused by fetal leukemia.

8 The first is leukemic cell involvement of multiple fetal organs and placenta that yields 9 excessive fluid accumulation in the fetal body and polyhydramnios. In this case, 10 multiple organ failure due to leukemic dissemination and/or acute circulatory failure 11 due to placental vessel occlusion may lead to sudden fetal demise [9]. The second is 12 chronic circulatory insufficiency due to hyperviscosity attributable to leukocytosis, 13 which is often accompanied by oligohydramnios. Hydrops fetalis in the second 14 situation often takes a slower course than that in the first situation [7, 8].

15 Prenatal diagnosis of congenital leukemia requires cordocentesis. According to our 16 PubMed search, prenatal diagnoses have been reported in only 10 cases [4, 5, 7, 8, 11, 17 12]. Interestingly, all of these cases were associated with trisomy 21 (Down syndrome). This suggests that leukemic fetuses associated with Down syndrome are likely to have a milder prenatal course than those without Down syndrome, thus giving a time for cordocentesis. Supporting this, neonatal leukemia associated with Down syndrome has a natural tendency to undergo spontaneous remissions and reported mortality rate is much lower than that without Down syndrome (30\% vs. 80\%) $[13,14]$.

Leukemic fetuses, especially those without association of Down syndrome, usually require postnatal chemotherapy. Although several reports described successful chemotherapy against leukemia during the early neonatal period [15-18], these neonates were in relatively stable conditions before initiating the chemotherapy. Since most of the leukemic fetuses are likely to be already deteriorated in utero, the rescue might be difficult even if the prenatal diagnosis was established and the delivery was expedited before the fetal demise. Recently, accumulating experiences of chemotherapy against pregnant women complicated with malignancies suggest that in-utero exposure to at least some of chemotherapeutic agents in the second and third trimester is relatively safe

32 in terms of fetal malformation as well as both short- and long-term neonatal outcome 33 [19-21]. Therefore, intrauterine chemotherapy might be an option to rescue prenatally 
1 diagnosed leukemic fetuses.

2 
1 References

2

31 Bader JL, Miller RW: Us cancer incidence and mortality in the first year of

$4 \quad$ life. Am J Dis Child 1979;133:157-159.

52 Greaves M: Pre-natal origins of childhood leukemia. Rev Clin Exp Hematol $6 \quad 2003 ; 7: 233-245$.

73 Gray ES, Balch NJ, Kohler H, Thompson WD, Simpson JG: Congenital

8 leukaemia: An unusual cause of stillbirth. Arch Dis Child 1986;61:1001-1006.

94 Gaedicke G, Kleihauer E, Terinde R: Acute non-lymphocytic leukaemia

10 versus transient leukaemoid reaction in fetuses with down syndrome. Lancet $11 \quad 1990 ; 335: 857$.

125 Foucar K, Friedman K, Llewellyn A, McConnell T, Aisenbrey G, Argubright

$13 \mathrm{~K}$, Ballinger L: Prenatal diagnosis of transient myeloproliferative disorder via

14 percutaneous umbilical blood sampling. Report of two cases in fetuses affected by

15 down's syndrome. Am J Clin Pathol 1992;97:584-590.

166 Donnenfeld AE, Scott SC, Henselder-Kimmel M, Dampier CD: Prenatally

17 diagnosed non-immune hydrops caused by congenital transient leukaemia. Prenat Diagn 18 1994;14:721-724.

197 Baschat AA, Wagner T, Malisius R, Gembruch U: Prenatal diagnosis of a

20 transient myeloproliferative disorder in trisomy 21. Prenat Diagn 1998;18:731-736.

218 Robertson M, De Jong G, Mansvelt E: Prenatal diagnosis of congenital

22 leukemia in a fetus at 25 weeks' gestation with down syndrome: Case report and review

23 of the literature. Ultrasound Obstet Gynecol 2003;21:486-489.

249 Shibasaki T, Matsuda H, Kawakami Y, Furuya K: Fetal leukemia with

25 umbilical artery embolism and circulatory failure. Obstet Gynecol 2007;109:521-523.

2610 Simpson JH, McDevitt H, Young D, Cameron AD: Severity of non-immune

27 hydrops fetalis at birth continues to predict survival despite advances in perinatal care.

28 Fetal Diagn Ther 2006;21:380-382.

2911 Zerres K, Schwanitz G, Niesen M, Gembruch U, Hansmann M, Waldherr R:

30 Prenatal diagnosis of acute non-lymphoblastic leukaemia in down syndrome. Lancet 31 1990;335:117. 
112 Macones GA, Johnson A, Tilley D, Wade R, Wapner R: Fetal

2 hepatosplenomegaly associated with transient myeloproliferative disorder in trisomy 21.

$3 \quad$ Fetal Diagn Ther 1995;10:131-133.

413 Homans AC, Verissimo AM, Vlacha V: Transient abnormal myelopoiesis of

5 infancy associated with trisomy 21. Am J Pediatr Hematol Oncol 1993;15:392-399.

614 Isaacs H, Jr.: Fetal and neonatal leukemia. J Pediatr Hematol Oncol

$7 \quad 2003 ; 25: 348-361$.

815 Mori T, Kaneko H, Kumagai MA, Miyauchi J, Kaneko Y, Fujimoto J,

9 Tsunematsu Y: Congenital leukaemia with a mixed phenotype of megakaryoblasts and

10 erythroblasts: A case report and characterization of the blasts. Br J Haematol

11 1997;96:740-742.

1216 Fernandez MC, Weiss B, Atwater S, Shannon K, Matthay KK: Congenital

13 leukemia: Successful treatment of a newborn with $\mathrm{t}(5 ; 11)(\mathrm{q} 31 ; \mathrm{q} 23)$. J Pediatr Hematol

14 Oncol 1999;21:152-157.

1517 Satter EK, Maari CH, Morel KD, Eichenfield LF, Cunningham BB,

16 Friedlander SF, Bergman JN: Disseminated linear calcinosis cutis associated with the

17 koebner phenomenon in an infant with congenital acute monocytic leukaemia. Br J

18 Dermatol 2004;150:753-756.

1918 van Dongen JC, Dalinghaus M, Kroon AA, de Vries AC, van den

20 Heuvel-Eibrink MM: Successful treatment of congenital acute myeloid leukemia

21 (aml-m6) in a premature infant. J Pediatr Hematol Oncol 2009;31:853-854.

2219 Aviles A, Neri N: Hematological malignancies and pregnancy: A final report

23 of 84 children who received chemotherapy in utero. Clin Lymphoma 2001;2:173-177.

2420 Ring AE, Smith IE, Jones A, Shannon C, Galani E, Ellis PA: Chemotherapy

25 for breast cancer during pregnancy: An 18-year experience from five london teaching

26 hospitals. J Clin Oncol 2005;23:4192-4197.

2721 Hahn KM, Johnson PH, Gordon N, Kuerer H, Middleton L, Ramirez M, Yang

28 W, Perkins G, Hortobagyi GN, Theriault RL: Treatment of pregnant breast cancer

29 patients and outcomes of children exposed to chemotherapy in utero. Cancer 30 2006;107:1219-1226. 
1 Figure legends

2

3 Figure 1. Sequential changes of pulsatility indices of umbilical artery (UmA-PI) (A)

4 and middle cerebral artery (MCA-PI) (B) and ultrasonographic findings 12 hours prior

5 to fetal death (C and D)

6 (A, B) Arrows indicate the time of fetal death. Note acute elevation of UmA-PI and

7 decline of MCA-PI from 4 days before fetal death at $30^{+3}$ weeks' gestation. (C)

8 Amniotic fluid depth of $109.3 \mathrm{~mm}$ indicates polyhydramnios. (D) Echo-free space

9 around fetal lung (arrowheads) represents pleural effusion. * indicates fetal heart.

11 Figure 2. Fetal cardiotocograms showing fetal heart rate (FHR) tracing in the upper 12 panels and uterine contractions in the lower panels.

13 (A) FHR tracing 13 hours before fetal death shows decreased beat-to-beat variability.

14 (B) FHR tracing 4 hours before fetal death shows small dip-like deceleration on every 15 uterine contraction. (C) FHR tracing immediately before fetal death shows mild 16 bradycardia (100-110 beat per minute) with loss of variability followed by sudden 17 disappearance of FHR. Arrow indicates the time of fetal death.

19 Figure 3. Placenta with fetal vessels showing leukocytosis. Scale bars indicate 100 $20 \mu \mathrm{m}$.

21 (A) Hematoxylin and Eosin (H\&E) staining. (B) Magnified image of the boxed area 22 shown in (A). Intravillous capillary vessels (arrows) are filled with small- to 23 medium-sized immature-looking atypical cells. (C) H\&E staining. Umbilical artery 24 (arrowheads) contains an aggregate of the atypical cells. (D) Myeloperoxidase 25 immunohistochemistry. Cytoplasmic staining of the atypical cells indicates myeloid 26 lineage of derivation. (E) Ki-67 (MIB-1) immunohistochemistry showing high 27 proliferative capacity of cells. Note that the labeling index reaches $50 \%$. 
2 京都大学1
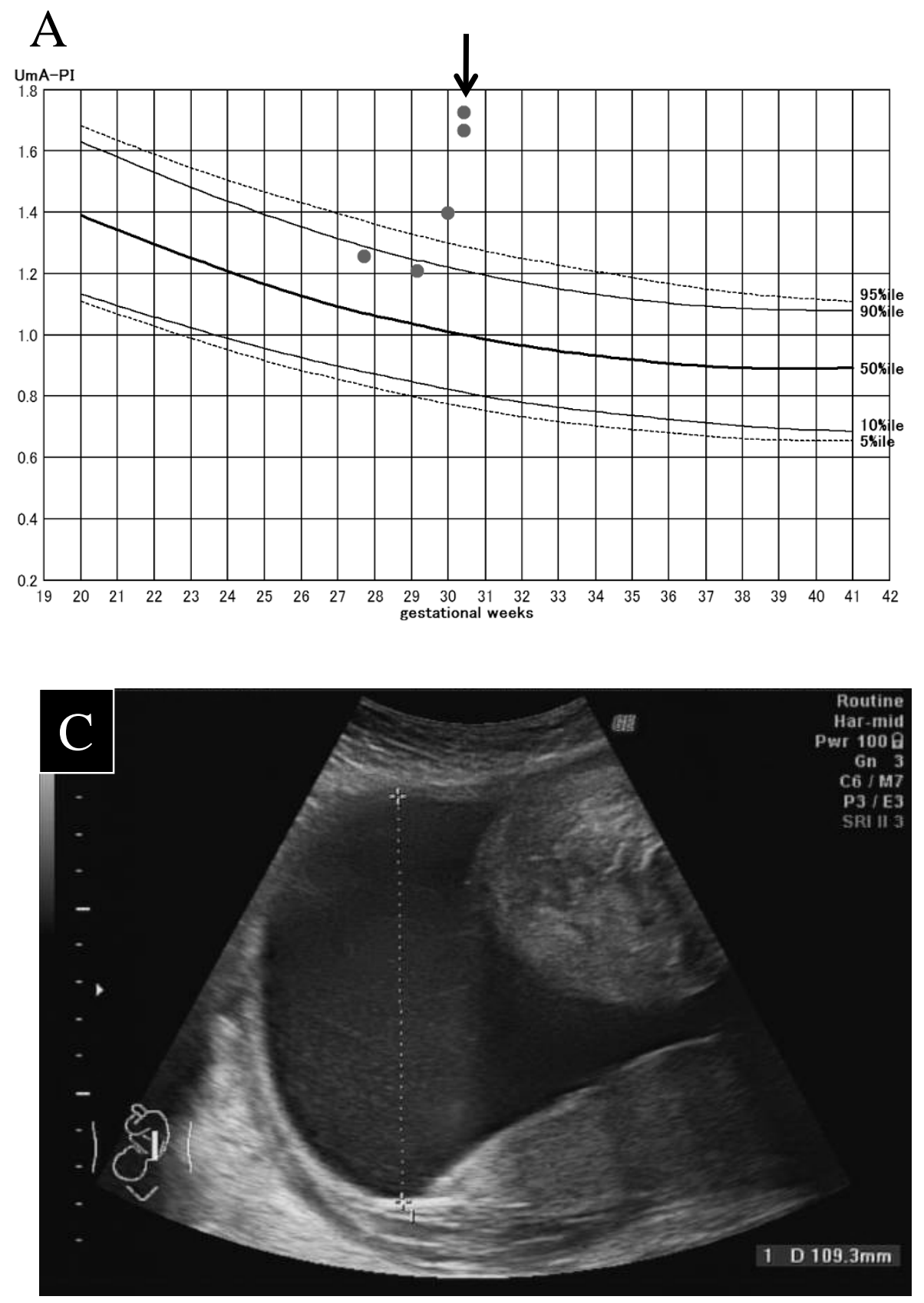
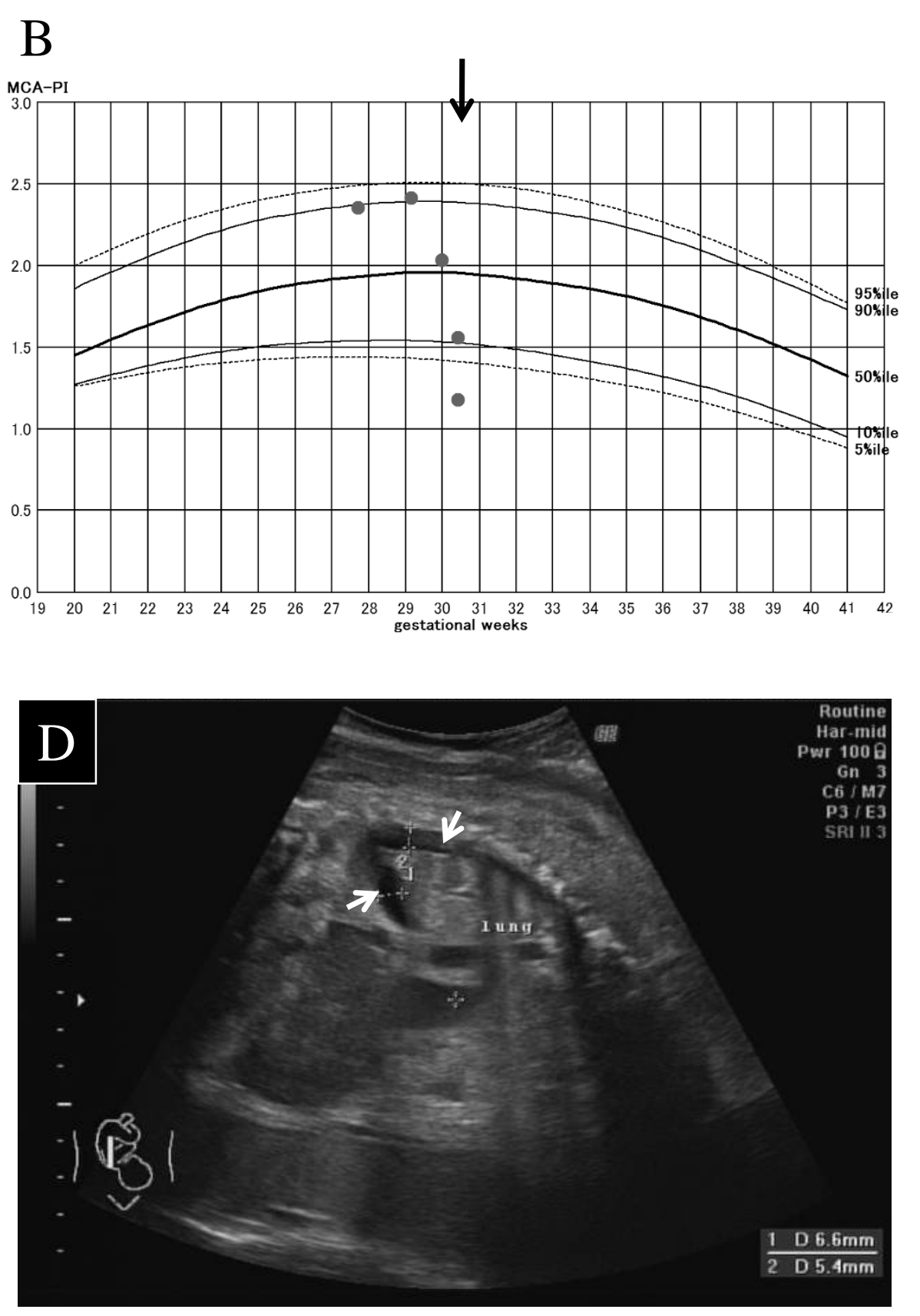

A
B
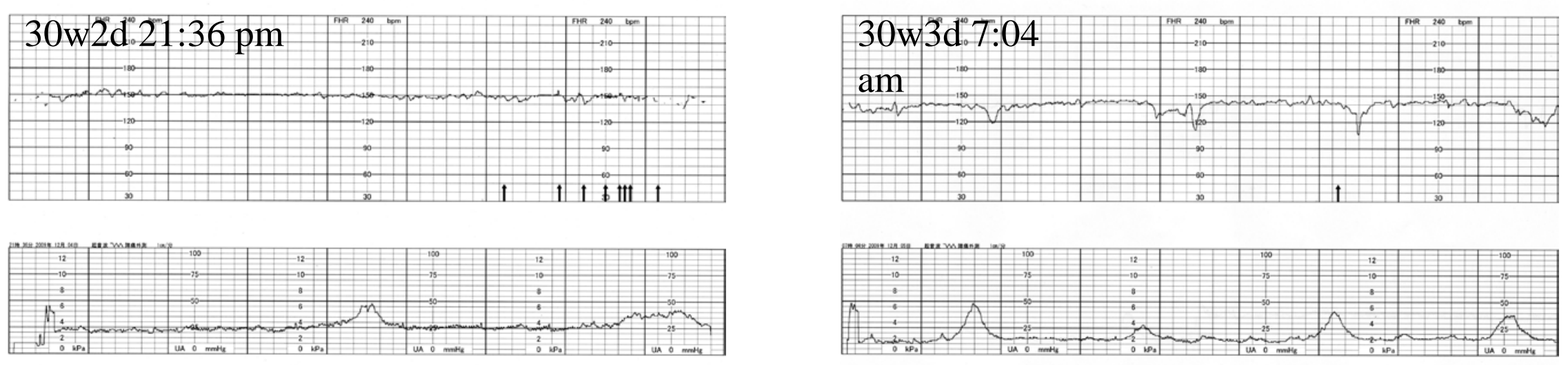

C

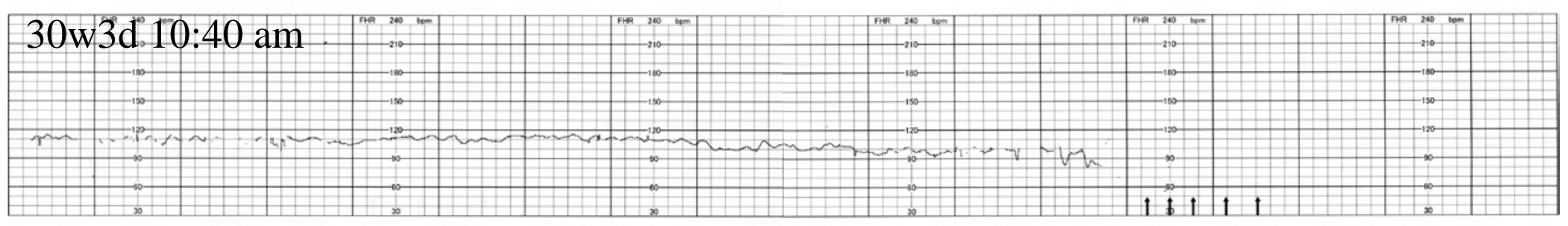

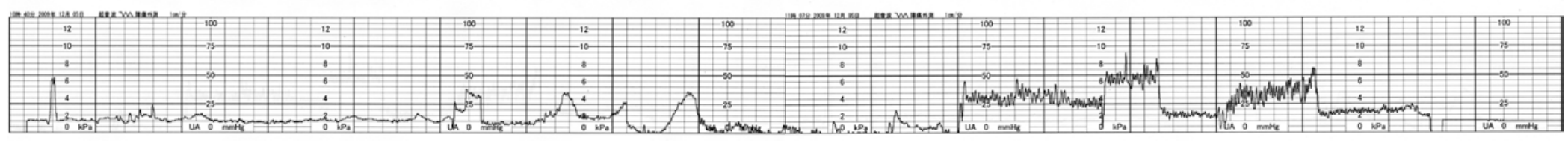



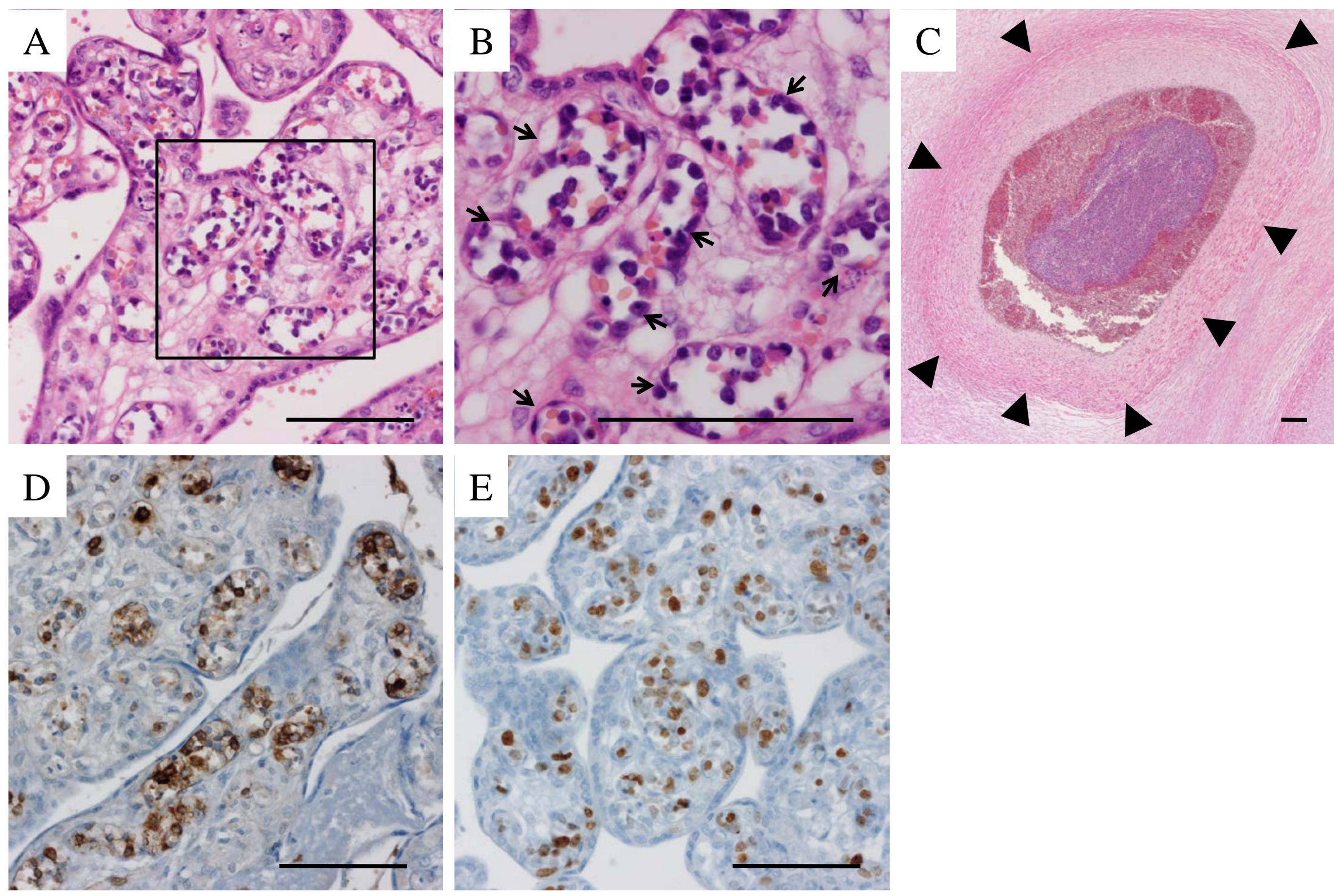